Stoa,

Vol. 1, No. 2, 2010, pp. 61-77

ISSN: $2007-1868$

\title{
DE LA FILOSOFÍA DE LA TÉCNICA A LA FILOSOFÍA COMO METATÉCNICA
}

\author{
JAIME FISHER \\ Instituto de Filosofía \\ Universidad Veracruzana \\ jaime.fisher@gmail.com
}

'[L]a falta de responsabilidad filosófica
de una década puede convertirse en la
tragedia política real de unas décadas
más tarde. Y la deconstrucción sin
reconstrucción es irresponsabilidad.'
Hilary Putnam: Cómo renovar la filosofía

RESUMEN:Se intenta transitar desde una reflexión sobre la técnica hacia una concepción de la filosofía misma como la técnica que permite evaluar toda acción humana en el mundo, i. e. como metatécnica. A la vez, se intenta sostener que hoy esa metatécnica ha de ser entendida como filosofía política.

PALABRAS CLAVE:técnica, filosofía, política, racionalidad.

SUMMARY:In this paper I try to come from the philosophical reflection on technique to the concept of philosophy as the technique that allows us to evaluate every human action, i. e., as meta-technique. At the same time I try to sustain that political philosophy must be seen, precisely as that meta-technique.

KEY WORDS:technique, philosophy, politics, rationality.

0 . El objetivo del ensayo es proponer y fundamentar la necesidad práctica de entender a la filosofía como la actividad humana que hoy resulta más importante y urgente ejercitar y mejorar. Pretendo mostrar esto al transitar desde una comprensión filosófica de la técnica, i. e., de la acción humana sistemática intencional, hacia el entendimiento de la filosofía como esa técnica particular que se incluye recursivamente como objeto de su reflexión. Se concluirá que si hubiera una filosofía primera, ésta debería hoy enten- 
derse como filosofía política y no como metafísica, en ninguno de los sentidos que esta multivalente y polisémica palabra suele tener. El apartado 1 intenta precisar el objeto de la filosofía de la técnica. El apartado 2 plantea a la ciencia, la tecnología, la economía, la política y a la filosofía misma como instancias particulares de la técnica. En el apartado 3 argumento cómo la filosofía es una metatécnica y por qué es inaplazable llevarla a cabo hoy qua filosofía política.

1. En un sentido amplio la técnica puede considerarse como la manera específicamente humana para meter cierto orden en el mundo, ese orden mínimo sin el que toda práctica vital (sistemática e intencional, $i$. e., técnica) resultaría imposible. De ahí se infiere que tecnicidad y humanidad son coextensivas, es decir, que no exista la técnica sin el hombre ni el hombre sea posible sin la técnica, pues no hay orden humano al margen de ésta. ${ }^{1}$

La filosofía misma ha considerado esta búsqueda primordial del orden sólo de una manera implícita, y ella misma, como intento técnico de meter orden al mundo de los conceptos, ha enfrentado el tema de la técnica a lo largo de la historia con mayor o menor éxito, desarrollando ahí algunas de las acepciones fundamentales del término que, no obstante, parecen por sí mismas insuficientes para precisar filosóficamente la naturaleza y el significado actual de la técnica. Entre tales acepciones destacarían las siguientes: a) un conocimiento o saber cómo (acepción básica compartida en general por los tratadistas), b) una acción intencional que implica un saber hacer (Bunge, Durbin, Mitcham, Rapp), c) un artefacto o conjunto de artefactos producidos y/o utilizados (Aristóteles, Kapp, Munford), d) un proceso socio-histórico manifiesto en el progreso (Bacon, Marx, V. Bush), e) un sistema complejo (Bijker, Ropohl, Quintanilla), f) la práctica distintivamente humana (Ortega, Gehlen, Cassirer).

Estos usos conceptuales no se excluyen, sino más bien se complementan entre sí. Cada uno de ellos hace referencia a distintos aspectos o dimensiones del mismo asunto $\mathrm{y}$, vinculados, bien pueden ayudar a precisar de qué hablamos cuando hablamos de técnica. El inciso (a) revela un saber o habilidad particular referida a cómo producir -o evitar que se produzcan- ciertos cambios en los estados o en los procesos de la realidad; un conocimiento acerca de cómo alcanzar ciertos objetivos. En esta acepción se manifiesta un primer aspecto epistemológico de la técnica; y dado que tal 'conocimiento cómo' una vez puesto en operación implica (b) la acción intencional, entonces ese conocimiento será un saber cómo hacer algo concreto en el mundo, acepción que destaca su dimensión praxiológica. Ya en su etimología original techné refiere a la observancia de ciertas reglas de las que depende la eficacia de la acción. En esas reglas

\footnotetext{
${ }^{1}$ Por orden se entiende aquí ciertas condiciones a partir y a través de las cuales los seres humanos son capaces de dirigir su acción intencional. El orden es entonces una cierta disposición de las cosas, realizada intencionalmente sobre algún aspecto del mundo físico o simbólico. El orden intencional es un resultado emergente de una transacción más básica, una de carácter orgánico-ambiental meramente biológica y que, sin embargo, puede ser ya entendida como conocimiento.
} 
-proposicionales o no- se manifiesta un saber cómo hacer ese algo concreto en que consisten oficios y artes técnicos, es decir, en cómo producir (o evitar que se produzca) un particular estado de cosas, un cierto estado ordenado en al menos algunas de las cosas que constituyen el mobiliario del mundo. Es en y a través de este vínculo praxiológico con la producción (poiesis) que la técnica da origen a (c) lo artificial y fenoménicamente se plasma en el artefacto material o simbólico, en eso cuya causa eficiente es un ars. De aquí que la técnica nos aparezca - parafraseando a Marx- como un inmenso arsenal de artefactos. Esta inmediata dimensión o apariencia fenoménica, supone el aspecto praxiológico que, a su vez, implica la dimensión epistemológica de la técnica. Entonces, así entendida en su nivel más básico y general, la técnica es una acción o conjunto de acciones intencionales sistemáticas, conducidas por cierto conocimiento acerca de la construcción y/o uso de artefactos para producir o evitar ciertos cambios en la realidad material o simbólica, es decir, para generar ahí cierto orden. Ahora bien, cuando a ese saber cómo hacer-propio de la técnica en sentido general— , se incorpora además un saber por qué la acción y el seguimiento de las reglas se asocian regularmente (causalmente) a sus efectos, nos hallamos frente a la tecnología propiamente dicha. La tecnología es pues la instancia de la técnica conducida por el conocimiento científico. En otras palabras, el fundamento y dimensión epistemológica de la tecnología se halla en la ciencia. ${ }^{2} \mathrm{Al}$ concepto general de técnica suele dársele en su uso cotidiano un significado ligado a éste particular de tecnología. Sin embargo, como se infiere de lo anterior, estos términos no son sinónimos: mientras que la técnica es un saber cómo hacer algo, la tecnología es una técnica de base científica, es decir, una técnica en la que alguien sabe por qué y cómo ciertos efectos son regularmente producidos en la acción considerada. Aunque toda tecnología es una técnica no se cumple la inversa. El término tecnología pretende indicar y reflejar un mayor grado de control sobre la acción y sus resultados. Podemos, por tanto, usar adecuadamente el término más extenso de técnica para referirnos a la transformación intencional y sistemática de objetos materiales o inmateriales, y a ambas clases de saber hacer ( saber cómo hacer que ocurran ciertas cosas, y saber por qué esas cosas ocurren como resultado de la acción). Este concepto amplio de técnica abarca saber cómo hacer fuego a partir de dos piedras; saber cómo producir los medios materiales y culturales de vida; saber cómo hacer que un artefacto descienda sobre la superficie de Titán; saber cómo hacer cosas con palabras o símbolos; saber cómo evitar que los hombres se maten entre sí; saber cómo producir la justicia, y saber cómo llegar a conocer (ordenar) determinados aspectos de la realidad física, biológica o cultural. ${ }^{3}$

\footnotetext{
${ }^{2}$ Esto ha conducido equívocamente -a Mario Bunge entre otros- , a identificar la tecnología con la ciencia aplicada. Si bien la tecnología se fundamenta en la ciencia, no se reduce a ésta pues, como se verá, el conjunto de decisiones que se involucra en la tecnología abarca e implica asuntos que van mucho más allá del conocimiento generado por la ciencia.

${ }^{3}$ No todo orden conseguido técnicamente resulta praxiológicamente adecuado. Baste recordar al respecto la clasificación de los animales según la enciclopedia china citada por Borges en El idioma
} 
Un punto crucial aún no desarrollado en filosofía de la técnica, que hasta el momento ningún autor ha considerado y que yo intento incorporar expresa y cardinalmente en la agenda temática, consiste en que toda aplicación técnica expresa siempre un conjunto de elecciones y decisiones: 1) sobre la acción o acciones específicas deliberadas entre todas las que cabe hacer al hombre, 2) sobre los medios e instrumentos utilizables, 3) sobre los posibles objetivos concretos de la acción (qué cambios producir o evitar en la realidad, es decir, qué tipo de orden generar), 4) sobre los fines de la misma, i. e., sobre el para qué de la acción y de los cambios que pretende insertar en el mundo, buscando darle entonces a ese mundo un orden determinado en lugar de otro; y finalmente pero nunca al último 5) sobre los resultados que efectivamente obtenga y que, entonces, se decida también aceptar o rechazar. Las elecciones 1-5 constituyen ese sistema decisional en el que consiste la naturaleza de toda manifestación técnica. Como en todo sistema, la naturaleza y significado de éste son irreducibles a alguna de sus partes, i. e., no pueden ser adecuadamente descritos ni explicados ni comprendidos a partir de la descripción, explicación o comprensión de alguno de sus componentes aislados. De aquí deriva tanto una justificación lógica como, más importantemente, una conveniencia práctica de concebir el estatus ontológico de la técnica como ese conjunto sistémico de decisiones, cuya articulación es llevada a cabo por un agente, individual o colectivo, quien, si ha de ser racional, ha de considerar entonces el contexto físico-temporal y simbólico-cultural bajo el que la aplicación tenga lugar. En otras palabras, el carácter holístico o sistémico de la técnica como conjunto decisional implica que cada una de esas elecciones asume valoraciones que a su vez, si la técnica ha de ser racional, no podrán ser contradictorias o inconsistentes entre sí para el agente que las conduce, las evalúa y, basándose en ellas, actúa en un contexto espaciotemporal.

Incorporar y considerar esta ontología decisional en la reflexión filosófica sobre la técnica es crucial porque permite y conduce a dos cosas filosóficamente importantes y, según creo, de muy promisoria fertilidad. En primer lugar es que sólo viéndola como conjunto de elecciones y decisiones - por necesidad valorativas- la técnica muestra de manera plena su dimensión axiológica, faceta que intenta recogerse en (d) el concepto de progreso técnico que, en un sentido lógico estricto, sólo puede interpretarse como el hecho de que el resultado final de la práctica ( el sistema total de cambios producidos y ordenados en, por y a través de las decisiones) sea valorado positiva y razonablemente. Y por ello, en segundo lugar en cuanto a su aparición y en primer lugar en cuanto a su importancia, en que esa misma ontología decisional básica permite y conduce a resaltar el papel central de la razón en el diseño, aplicación y evaluación de la técnica y, 
así, a enfocar y precisar la relación entre los conceptos de racionalidad y progreso. ${ }^{4}$ Entre las cosas que tal ontología permite hacer destaca disolver la falsa dicotomía tecnofiliatecnofobia, centrando el tema hoy fundamental de la evaluación de la técnica ${ }^{5}$ a partir del ejercicio de la razón humana: la técnica no está pre-determinada ni pre-destinada, ni optimista ni pesimistamente al bien ni al mal, pues depende de las elecciones y decisiones que a cada paso sea capaz de tomar - y en su caso corregir- , el ser humano. No está de más señalar al respecto la sugerencia orteguiana en relación al presunto origen etimológico común de elegir, elegancia e inteligencia: se sigue que si la técnica es ontológicamente algo elegido en libertad, entonces la elegancia y la inteligencia de la técnica dependerán del orden y bienestar ${ }^{6}$ que produzcan para los seres humanos que la diseñan, la ejecutan y se exponen a sus resultados. En este sentido la inteligencia coincidiría con la racionalidad ${ }^{7}$, es decir, con cierta ratio ( sentido de la proporción) entre las diversas elecciones y decisiones constitutivas de cada sistema técnico específico, así como con cierto orden entre todos los sistemas técnicos activos en un determinado contexto de operación.

Todas estas dimensiones y aspectos de la técnica (epistemológica, praxiológica, fenoménica, ontológica y axiológica) se imbrican en una relación estrecha y cambiante, cuya naturaleza y significado específicos puntuales hay que esclarecer como objetivo central al plantear el tema de su evaluación desde la perspectiva filosófica. Esto es lo que hace de la filosofía de la técnica una (meta) técnica, y, en este caso, esa técnica que tiene por objeto poner de manifiesto la naturaleza y significado de la técnica en general, incluyéndose ella misma en el objeto de estudio. ${ }^{8}$

${ }^{4}$ Es por esto que en la noción de progreso parece centrarse el asunto de la evaluación de la técnica. Tal noción se vincula con la racionalidad como eventual característica en el uso práctico de la razón humana. Hay aquí un concepto filosóficamente discutible de razón y de racionalidad en el que no puedo detenerme.

${ }^{5}$ Como más adelante se afirma, la ciencia, la tecnología, la economía, la política y la filosofía misma son las instancias de la técnica con mayor importancia contemporánea.

${ }^{6}$ El contenido del bienestar suele ser muy distinto para diversos individuos, e incluso para un individuo en el mismo o en distintos momentos de su vida. Por bienestar entenderé en un sentido amplio a la conjunción de los valores políticos fundamentales de justicia en la organización social y libertad de sus individuos, es decir, las condiciones mínimas a partir de las que cualquier ciudadano pueda dar un contenido específico a su concepto particular del bien y del bienestar. La tolerancia se implica lógicamente entre los valores políticos.

${ }^{7}$ Rescher aboga en este mismo sentido al definir la racionalidad como el uso inteligente de la razón.

${ }^{8}$ Cambios en el conocimiento conducen a cambios en las prácticas, en los artefactos y en los valores disponibles para ser elegidos en una técnica. Los cambios en los valores parecen ser los más lentos del proceso y, en particular valores como la eficiencia instrumental y la rentabilidad, que conducen técnicas 
Ahora bien, si el cimiento ontológico de la técnica es, como propongo, ese conjunto de decisiones empíricamente visibles en una práctica, entonces la filosofía de la técnica es una filosofía de la acción, y más específicamente una filosofía de la acción humana sistemática intencional, misma que se manifiesta necesariamente en un particular artefacto: e) el sistema técnico ${ }^{9}$, esa unidad fenoménica básica sobre la que gravita la reflexión y crítica filosófica, en tanto que tal artefacto es considerado f) algo distintivamente humano, i. e., algo que permite distinguir al hombre del resto de los seres vivos. Es en ese sistema técnico en donde se manifiesta la técnica como conjunto de elecciones y decisiones deliberadas. Más exactamente desde el punto de vista lógico y más importantemente desde el punto de vista axiológico, en tales elecciones y decisiones distintivamente humanas, es decir, en tal ontología, es donde se establecen aquellas potencialidades que constituyen al hombre, potencialidades que él mismo juzga valiosas en determinado contexto y que, sólo entonces, elige y decide intentar actualizar ${ }^{10}$ Conviene pues a la supervivencia y a la bienvivencia -individual y colectiva- revisar críticamente sus condiciones y resultados reales y potenciales, e intentar hacerlo desde la razón es lo mejor que cabe hacer. Tal es la tarea de la filosofía de la técnica.

2. Son cuatro los ámbitos civilizatorios de manifestación técnica donde hoy destaca la urgencia de esa reflexión: la ciencia, la tecnología, la economía y la política. El hecho que urge por esta deliberación son las condiciones y resultados del desenvolvimiento científico y tecnológico, en un contexto donde la política muestra cada vez menos eficacia para controlarlos y producir el bien común, y donde este bien común es imposible e impensable sin el bienestar

como la tecnología y la economía, siguen siendo básicamente los mismos hoy, pero bajo condiciones de desarrollo científico y tecnológico que permitirían hacer cosas razonablemente mejores, es decir, buscar valores que, como la justicia y la libertad, se hallan hoy al alcance de las elecciones humanas racionales.

${ }^{9}$ Un sistema técnico es trabajo conducido por conocimiento (científico o del sentido común, tácito o proposicional) dirigido a la transformación de objetos o situaciones concretas, materiales o inmateriales, asociado a un resultado convencional y tempo espacialmente limitado y definido considerado útil por el agente, Fisher 2010, pp. 105

${ }^{10}$ Entre las implicaciones lógicas de esto destaca la negación de todo determinismo y la afirmación de la libertad de la voluntad humana (free will). Sin embargo, no es este un tema en el que convenga detenerse. $\mathrm{Al}$ menos por el momento y en el contexto de este breve ensayo. 
económico entendido como ese cierto orden que permite el acceso a los bienes indispensables para sobrevivir e intentar bienvivir. ${ }^{11}$

Existen, por supuesto, relaciones claras entre la ciencia y la tecnología, entre estas dos y la economía, y de las tres con la política, pero cada una de ellas es distinguible por sus objetivos particulares. Podemos comprender la ciencia como una instancia o manifestación técnica cuyo objetivo es meter cierto tipo de orden en el mundo, cambiando ciertas parcelas de éste de desconocidas en conocidas, en el sentido de sujetas a explicación o comprensión científica. ${ }^{12}$ En esta técnica la dirección del cambio va fundamentalmente del mundo hacia la mente, pues el orden que intenta captar y producir la ciencia es uno que se halla condicionado centralmente por la estructura del mundo, estructura que intenta capturarse bajo la forma de leyes de la naturaleza, así sean estas de carácter estocástico. En la tarea de obtener tal orden la ciencia suele utilizar artefactos tecnológicos, al tiempo que el conocimiento así obtenido suele también incorporarse al diseño y construcción de nuevas aplicaciones tecnológicas. Esto ha dado pie al uso cada vez más extendido del término híbrido tecnociencia. Sin embargo, la tecnología en sentido estricto tiene por objetivo principal, más bien, la transformación de algún aspecto del mundo físico, biológico o simbólico con base en determinadas ideas o conocimiento científicos, por lo que ahí la dirección del cambio y del orden que se busca introducir corre fundamentalmente de la mente hacia el mundo.

Con economía no me refiero aquí a la disciplina de las ciencias sociales (economics) del mismo nombre en castellano, sino a la práctica intencional sistemática que tiene por objetivo la organización de la producción, distribución y consumo de los bienes materiales y simbólico-culturales necesarios para la vida humana (economy), y cuyos agentes organizadores se hallan motivados centralmente por la rentabilidad. ${ }^{13}$ Estas tres instancias de la técnica -en su

\footnotetext{
${ }^{11} \mathrm{El}$ concepto de renta básica es útil al respecto. Consistiría en un ingreso suficiente para la sobrevivencia, como un derecho más a los de ciudadanía, y sin importar nada más que esa cualidad de ser ciudadano.

${ }^{12}$ Estoy incorporando por supuesto en la noción de cambio al cambio de la clase llamada Cambridge change, un cambio que rebasa por mucho el de la materia o el de los cambios actuales, para incorporar tanto a lo intencional como a lo meramente potencial: un mundo en el que hay conocimiento o intenciones es distinto a un mundo en donde no los hay.

${ }^{13}$ Este concepto requiere algunas precisiones. Bajo el sistema de libre mercado, la rentabilidad es entendida cuantitativamente como una relación entre el volumen de recursos comprometidos técnicamente en la producción y el volumen de recursos obtenidos de su realización o venta. Sin embargo, este con-
} 
búsqueda de orden - ocurren en un contexto histórico y cultural con el que interactúan. Sin dejar de considerar campos de importancia civilizatoria como la religión o el arte, parece hoy conveniente focalizar la atención en la política. Para decirlo pronto, entenderé por política en un sentido estricto a esa técnica cuyo objetivo es aquel cierto orden social que desde la antigüedad se ha llamado justicia. Deben por ello dejarse aquí fuera de las notas distintivas de política aquéllas acepciones del término que, dada su polisemia en el habla cotidiana en nuestro idioma, resultan particularmente confusas y equívocas. Suele usársele como: a) acción humana gregaria fundamental en busca del bien común (la praxis aristotélica), b) como una acción específica de gobierno (la 'política' económica), c) como régimen (la 'política' mexicana), d) como habilidad para la adquisición y ejercicio de algún tipo de poder (no necesariamente estatal), es decir, como negociación y/o intriga, o lo que en el castellano de México se llama grilla; estrechamente vinculado a este uso también suele concebirse una acepción coloquial 'maquiavélica', e) como lucha descarnada por el poder; f) como estrategia de empresas o particulares (la 'política' de compras); g) como sustantivo (Beatriz Paredes es una 'política'); h) como comportamiento en ámbitos que pueden ser estrictamente privados ('corrección política'); i) como rama disciplinar de estudio (la filosofía o la ciencia política); y hasta j) como título de la obra seminal de la filosofía aristotélica: La Política. En esta polisemia del término se origina que se le suela usar indistintamente y respecto de prácticas no necesariamente estatales (la 'política' estudiantil, sindical, académica, o incluso familiar, etc.), no necesariamente públicas, no necesariamente de interés para un colectivo humano, y no necesariamente con el objetivo de la justicia. Esto presenta el primer problema básico bajo la forma de establecer también claramente de qué hablamos cuando hablamos de política, asunto que bien merece una breve reflexión.

Si acotamos y restringimos aquí el uso de política para la primera acepción listada, misma que se rescata en el término inglés politics o statecraft, y que en

cepto no es capaz de capturar las externalidades del proceso de producción, distribución y consumo que se generan sobre el medio ambiente físico y, sobre todo, sobre el simbólico-cultural. No se cuenta con un concepto que sí lo haga. La contabilidad del producto interno bruto ecológico, por ejemplo, es un intento al respecto, sin embargo, éste considera sólo las externalidades físicas y deja de lado a las simbólico-culturales que suelen ser mucho más importantes. En otro sitio he propuesto el concepto cualitativo de eficiencia sistémica, que puede sustituir adecuadamente a éste de la mera rentabilidad cuantitativa. 
dicho idioma se distingue de otros como polity (organización social no necesariamente política o estatal), policy (estrategia o vía de acción a seguir, no necesariamente estatal o colectiva), political como adjetivo aplicable tanto al hacer público como al privado, y politician (político o política) como sustantivo; entonces esa política queda precisada - en su sentido de técnica- , como esa praxis que, en esta acepción original, tiene por objetivo la producción de justicia o el bien común más básico y elemental.

Amerita detenerse brevemente aquí porque la justicia es el asunto público nodal del estado o del sistema político qua sistema técnico y, como dice Perelman (1964, p. 15), 'entre todas las nociones prestigiadas, la justicia parece una de las más eminentes y la más irremediablemente confusa'. En este mismo tenor Kelsen afirma que

'[S] obre ninguna otra pregunta han meditado más profundamente los espíritus más ilustres -desde Platón a Kant- . Y sin embargo, ahora como entonces, carece de respuesta. Quizá sea porque es una de esas preguntas para las cuales vale el resignado saber que no se puede encontrar jamás una respuesta definitiva sino tan sólo preguntar mejor'. (Kelsen 1995, 11)

Por supuesto, no se pretende aquí producir un concepto de justicia que resuelva, de una vez por todas y al gusto de todos los filósofos de la política y del derecho, la añeja discusión al respecto, sino sólo y precisamente preguntar mejor. Y preguntar mejor hoy parece cruzar por vincular sistémicamente el concepto de justicia, objetivo propio de la política y del sistema político (Estado), con los conceptos de verdad, eficiencia y rentabilidad, valores propios de los sistemas técnicos en la ciencia, la tecnología y la economía, respectivamente; viendo a esas cuatro prácticas y a sus objetivos como las instancias hoy fundamentales de la técnica, de esa práctica con la que de hecho el hombre responde a la pregunta acerca de cómo vivir.

Puesto que esta pregunta implica ya una respuesta en el sentido de la vida gregaria y cooperativa, podemos entender directa y simplemente por justicia al concepto clásico de dar a cada quien lo que sea debido, cosa que implica el no dar a alguien lo que no sea debido, y en este particular sentido, el no producir algún tipo de violencia física o simbólica injustificada a través de un medio ambiente irremediablemente compartido, en condiciones de recursos escasos, y de intereses, cosmovisiones y creencias individuales distintas y muchas veces contrapuestas entre sí. 
Este concepto naturalista de justicia presupone e implica lógicamente cierta noción igualmente naturalista de libertad. Los individuos - en condiciones de justicia o equidad en las relaciones sociales-, serían libres respecto a otros ciudadanos y frente al estado para llevar a cabo aquellas acciones que ellos mismos consideraran valiosas y que no produzcan algún tipo de violencia injustificada, directa o indirecta, sobre otros ciudadanos. La política es entonces la técnica que se habría de ocupar de producir tanto la justicia del orden gregario como la libertad de sus individuos, pues si bien la libertad sin la justicia es ciega, la justicia sin la libertad es vacía y carente de sentido. Es en este contexto argumentativo que la filosofía política resulta ser la metatécnica que tiene por objetivo ese particular estado de cosas u orden que consiste en entender lo que la política qua técnica produce, lo que no produce y lo que evita producir, y así, a establecer conceptualmente los criterios empíricos de la medida en que se acerca o no a los valores centrales de justicia y libertad que idealmente esa técnica debería producir, valores políticos que se encuentran en una relación inestable respecto a la persecución de otros valores técnicos, como la verdad científica, la eficiencia tecnológica y la rentabilidad económica.

El asunto básico a que me refiero (la necesidad de la metatécnica ${ }^{14}$ ), y que hace necesario ver a la ciencia, la tecnología, la economía y la política como técnicas, consiste en las condiciones y resultados negativos del desenvolvimiento científico, tecnológico y económico sobre la justicia entre individuos, clases, regiones y naciones, y sobre la libertad de los individuos. El orden particular buscado y eventualmente obtenido por cada una de esas instancias técnicas puede resultar ser verdadero o falso, eficiente o ineficiente, rentable o desastroso, justo o injusto, esclarecedor o equívoco. La filosofía, al reflexionar sobre las condiciones y resultados de la técnica, adquiere entonces simultáneamente tanto el carácter de metatécnica como el de filosofía política. Parece este un camino interesante y fértil de cómo renovar la filosofía, que es la manera en que

\footnotetext{
${ }^{14}$ Una filosofía primera no puede ser entendida hoy en el sentido aristotélico. 'Si no existiese ninguna otra entidad fuera de las físicamente constituidas, la física sería ciencia primera. Si, por el contrario, existiese alguna entidad inmóvil, ésta sería anterior y filosofía primera, y será universal de este modo por ser primera.' (Aristóteles: Metafísica, VI, 1; Gredos, Madrid). Es claro que el sentido en que después se llamó a eso metafísica es un sentido muy otro al que podemos llamar hoy a la filosofía política una filosofía primera o metatécnica. Este carácter primero de la filosofía política no sólo es de carácter práctico sino también lógico. Un argumento amplio al respecto puede encontrarse en Smith 2007.
} 
Putnam pone en 1994 la preocupación de Dewey, en 1920, por la reconstrucción de la filosofía.

Recapitulando hasta aquí: la ciencia, la tecnología, la economía, la política y la filosofía misma, serían instancias de la técnica que buscan algún tipo de orden en el mundo como condición mínima básica del dar una respuesta a cómo se haya de vivir. Pero, como ya se apuntó, de hecho esas instancias de la técnica -y la técnica en general-, son en sí mismas ya una respuesta o, al menos, un intento de respuesta a tal pregunta. Desde un punto de vista lógico, plantearse la interrogación acerca de cómo vivir implica estar vivo y, por tanto, haberle dado ya alguna respuesta. Por tanto, la pregunta socrática no podría referirse al simple hecho de cómo hacer para vivir, sino más bien al de cómo se puede y se debe vivir bien, es decir, de acuerdo al bien. Pero en este caso, por ejemplo, la idea del bien, postulada metafísicamente por Platón en La República como condición de posibilidad y de 'perfección' de las demás Ideas, no puede sostenerse hoy, y no puede sostenerse por las mismas razones por las que no se puede sostener hoy la idea aristotélica de metafísica como filosofía primera, a saber, por considerar los resultados del avance en las ciencias, particularmente en la física, en la biología y en la neurociencias. O, en otras palabras, si bien la ciencia no se plantea problemas metafísicos, la filosofía no puede plantearse sus problemas (metafísicos o no) al margen de los problemas y soluciones planteados por la ciencia.

Esto es lo que requería - tanto en tiempos socráticos como hoy- de una reflexión, pues en ese entonces, como en este hoy, una vida sin reflexión no vale la pena de ser vivida. La filosofía práctica era entonces y es hoy esa reflexión fundamental acerca de lo que el hombre hace en la naturaleza. En este sentido la filosofía práctica (moral y política) nos aparece claramente como la meta-técnica, es decir, como ese hacer reflexivo intencional y sistemático sobre las condiciones y resultados que tiene la respuesta científica, tecnológica, económica y política al asuntillo ese acerca de cómo vivir.

3. Qua técnica o, en este caso más exactamente, qua meta-técnica, la filosofía busca también algún tipo de orden en el mundo, y, más exactamente en el mundo de las ideas y los conceptos que sirven al pensamiento que, a su vez, sirve como guía de la acción. La filosofía como pensar recursivo sobre sí mismo presupone, lo mismo que cualquier otra técnica, un conjunto de elec- 
ciones y decisiones sobre los objetivos, los instrumentos, los fines y los resultados (dimensión ontológica); se manifiesta en artefactos simbólico-lingüísticos (dimensión fenoménica); es conducida por cierto conocimiento y produce nuevo conocimiento (dimensión epistemológica); trata de hacer algo concreto, a saber, introducir cierto orden en el mundo (dimensión praxiológica); y presupone y genera esquemas valorativos (dimensión axiológica). De la misma manera en la que quien adopta una técnica adopta sus resultados sobre el orden que busca, así también quien adopta una filosofía adopta sus resultados sobre el orden pretendido. Como ya se apuntó líneas arriba, no todo orden resulta un orden bueno o aceptable desde el punto de vista praxiológico, y de ahí la necesidad de elegir entre técnicas filosóficas, es decir, entre filosofías. Dadas las dimensiones de la técnica apuntadas en el apartado 1 es menester ahora precisar los sentidos en que esas dimensiones se presentan en la filosofía misma como meta-técnica. Comenzando por un posible y aceptable punto de partida, fenoménicamente puede afirmarse que la filosofía nos aparece, en primer lugar, como un inmenso arsenal de artefactos simbólico-culturales, i. e., como un conjunto de preguntas, ideas y respuestas a varios asuntos que pueden razonablemente agruparse (clasificarse u ordenarse) en cuatro: 1) qué es el mundo; 2) qué es el hombre; 3) qué hace el hombre en el mundo; y 4) qué debe o debería hacer el hombre en el mundo una vez observados los resultados de sus respuestas empíricas a 1, 2 y 3 . Tales respuestas, a lo largo de la historia de la filosofía, producen y muestran cierto orden que puede ser, a su vez, más o menos eficaz (como un mapa) para orientar la práctica humana de supervivencia y de bienvivencia. No existe en este sentido, $L A$ filosofía, como no existe $E L$ mapa. Qué filosofía adoptar, seguir o producir dependerá, en todo caso, de las necesidades del filósofo, sea éste profesional o no; de la misma manera que el mapa a utilizar, seguir o producir dependerá de las necesidades efectivas sentidas por el viajero. Otra cosa sería ver la razonabilidad de las necesidades efectivamente sentidas por el filósofo en un momento y lugar dado de la historia. ${ }^{15}$

\footnotetext{
${ }^{15}$ Un filósofo bien puede sentir efectivamente la necesidad de plantearse y resolver hoy, por ejemplo, el tema del determinismo en relación con la libertad humana. Si bien nadie puede esgrimir buenas razones para impedirle pensar, escribir y publicar al respecto, también es cierto que él mismo no podría dar buenas razones para sentir y sucumbir a esa necesidad efectiva, excepto que siente subjetivamente tal. necesidad.
} 
Aunque fenoménicamente la filosofía puede tener diversas manifestaciones artefactuales específicas en éste o aquél sistema filosófico, ontológicamente sigue siendo un conjunto de decisiones o elecciones humanas, tanto si la vemos como producto a utilizar como si se le ve como proceso técnico a llevar a cabo. En tal sentido, la razón, esa que lleva a cabo el acto de elegir, integra también constitutivamente a la meta-técnica. ${ }^{16}$

En cuanto a su dimensión epistemológica el conocimiento filosófico es, como toda transacción del hombre y su medio ambiente, un proceso y un producto de la práctica. Como el científico, el tecnológico, el económico, el político y el del simple sentido común, el conocimiento filosófico tendría la función de guiar eficientemente la práctica misma de la que surge. Tiene, pues, (el conocimiento filosófico) también una función de supervivencia y de bienvivencia y se halla, en tal sentido, en el mismo plano de importancia que el científico, el tecnológico, el económico, el político y el del sentido común. Este conocimiento filosófico puede entenderse de dos maneras no necesariamente excluyentes, aunque rara vez complementarias en la acción cotidiana de los filósofos. Por un lado se le ve como el conjunto de ideas e intentos de solución, aportados por autores, escuelas y tradiciones, en torno a los diversos temas y problemas que constituyen la filosofía, sentido en el que se entiende ésta como mera erudición; y, por otro, como un particular hacer, como una cierta habilidad para demarcar los problemas, pasados o presentes, pensar y discurrir acerca de ellos - planteando nuevos problemas o revisando los antiguos desde otra perspectiva- ; caso éste en el que es un conocimiento práctico en el señalado sentido de saber hacer cosas con las ideas y con las palabras, sean estas propias o ajenas. Es aquí que la filosofía aparece muy claramente en su dimensión praxiológica como una labor o un saber hacer artesanal, una techné o un ars, más o menos simple o alambicada según sea el paladar del filósofo que la produce o que la consume, pero cuyos insumos son siempre las razones - en su acepción de ideas- , y su instrumento básico es la razón, en su acepción de capacidad cerebral de cómputo y procesamiento de información. Se supone también -muy

\footnotetext{
${ }^{16}$ Que una filosofía siempre se elija significa precisamente que las filosofías nunca valen lo mismo, que es lo opuesto a la conclusión de que todo vale como un corolario relativista y posmoderno de la irremediable participación del sujeto. Las filosofías, como cualesquier otras técnicas, resultan ser elegibles y elegidas porque resultan adecuadas o inadecuadas; esta adecuación o inadecuación se establece de manera convencional pero no de manera arbitraria.
} 
optimistamente por cierto- que el producto filosófico sea ese particular tipo de orden llamado entendimiento, es decir, una cierta disposición intencional de un conjunto sistémico de ideas, conexiones e inferencias, a partir del cual orientar eficazmente una respuesta a $L a$ pregunta, o al menos a una de sus preguntas afluentes. Con todo esto quiero decir que el conocimiento filosófico, ha de producir el entendimiento de uno mismo en el mundo al constituir un conjunto coherente de respuestas a preguntas como qué es el mundo, qué es el hombre, y qué hace y debe hacer el hombre en el mundo, preguntas que requieren al menos algunas respuestas fiables como condición para decidir cómo se haya de vivir.

Es al reflexionar sobre las condiciones y resultados actuales de la técnica -y principalmente de la ciencia, la tecnología, la economía y la política-, que la filosofía aparece como esa meta-técnica, cuyo objetivo es el entendimiento de lo que el hombre hace en relación a la supervivencia y de lo que debería hacer en torno a la bienvivencia. En particular, cuando se reflexiona sobre las condiciones y resultados de la ciencia, la tecnología y la economía, surgen de inmediato cuestiones de naturaleza moral que tienen que ver precisamente con la libertad y la justicia, valores fundamentales de la política en su sentido estricto aquí asumido. La dimensión pública ${ }^{17}$ de las aplicaciones científicas, tecnológicas y económicas, constituye el ámbito central de la reflexión práctica, que es entonces claramente una filosofía política, esa metatécnica o 'filosofía primera' a partir de la que puede comenzar a evaluarse la respuesta racional a la pregunta sobre cómo se haya de vivir. Esta es la dimensión axiológica de la metatécnica.

La filosofía política se pregunta sobre la legitimidad, racionalidad o justificación del estado y, desde hace mucho, ha llegado a la conclusión -compartida ampliamente entre los filósofos-de que un estado es legítimo si y sólo si produce el bien común, el orden social mejor, valga decir, la justicia. De ahí que la existencia o inexistencia de este orden justo sea el baremo que permite distinguir entre un estado y una simple banda de ladrones. Conforme

\footnotetext{
${ }^{17}$ Lo público se opone a lo privado, entendiendo por tal oposición no a la existente entre lo que sea del conocimiento público y la secrecía, ni a la existente entre el acceso público y la exclusividad individual o grupal, sino concretamente a la oposición entre los resultados causalmente derivados de una práctica o de una técnica, cuando, por un lado, estos se reducen a sus agentes directos y, por otro, cuando alcanzan a quienes no lo sean.
} 
los resultados del desenvolvimiento científico, tecnológico, económico y político se van desplegando, se van haciendo cada vez más amplios sus efectos negativos sobre la justicia de las sociedades y la libertad de la mayor parte de sus ciudadanos. La filosofía en general como metatécnica se ve así obligada hoy a considerar los valores científicos, tecnológicos y económicos como la verdad, la objetividad, la eficiencia y la rentabilidad, desde la perspectiva del inseparable par de valores políticos de justicia y libertad. Estas condiciones problemáticas planetarias para la elaboración de una respuesta a la pregunta acerca de cómo se haya de vivir, hacen de la filosofía política esa metatécnica fundamental, y nos la presentan hoy como 'filosofía primera', es decir, como aquella que más urgente e importante resultaría llevar a cabo. Esta filosofía primera no sería desde luego una reflexión 'acerca del ser en tanto que es', sino una reflexión sobre el hacer humano en tanto que hacer intencional con resultados sobre sus propias condiciones para poder seguir haciendo y siendo. Es en este sentido que tal filosofía primera no sería, pues, una 'metafísica', sino una metatécnica, la técnica de segundo orden que se cuestiona sobre lo que hace y debe hacer el hombre en el mundo.

El resumen de lo que se ha dicho hasta aquí expresaría que la técnica o, si se quiere, más bien la tecnicidad, esa cualidad y posibilidad de ser técnico, es lo que caracteriza a la naturaleza (historia) humana. Toda práctica intencional sistemática intenta actualizar alguna de las potencialidades del hombre y constituye una respuesta a cómo vivir. La ciencia, la tecnología, la economía y la política son las instancias técnicas civilizatorias que más claramente contribuyen a esa respuesta concreta. De ellas, la política es la instancia que, teniendo por objetivo la producción de la justicia correctiva y distributiva, haría legítimas, aceptables y racionales tanto a las condiciones como a los resultados de la operación de las tres primeras y, con ello, de sí misma. El estado aparece entonces como ese artefacto o sistema técnico cuya legitimidad depende crucialmente de producir la justicia y la libertad, bien positivamente a través de la ciencia, la tecnología y la economía, o bien en forma negativa, a través del derecho, evitando que la operación de estas técnicas resulten en alguna injusticia o en la disminución de la libertad. En otras palabras, la legitimidad del estado depende de su capacidad o adecuación para permitir y producir la racionalidad de las demás técnicas, pues cualquier manifestación técnica es racional si 
y sólo si incrementa (o al menos no disminuye) la libertad de los individuos y la justicia de las sociedades en que tales individuos viven. Se sigue entonces que la filosofía política es la metatécnica que permitiría producir el requerido control racional sobre la calidad del estado, en tanto que éste es visto como ese sistema técnico cuyos objetivos de libertad y justicia son perseguidos a través del control de calidad de los otros tres conjuntos de sistemas técnicos que hoy resultan más importantes, a saber, la ciencia, la tecnología y la economía. ${ }^{18}$

Así, en relación al objetivo de adecuar la filosofía a las exigencias de nuestro tiempo, no sólo es moral y políticamente irresponsable la deconstrucción relativista, como afirma Putnam en el epígrafe, sino también lo es la mera erudición de filósofo anticuario. Pero, sobre todo, la ignorancia -en el sentido doble de desconocimiento y de falta de consideración-, tanto por la historia de la filosofía que hoy resulta relevante, como por los resultados de la ciencia y la tecnología que tienen que considerarse filosóficamente para intentar responder a La Pregunta. Esa reconstrucción de la filosofía hoy bien podría intentarse, a partir de lo aquí apuntado, como una reconstrucción naturalista en la filosofía política. Tal vez valga proponer que la reconstrucción de la filosofía que necesitamos es más exactamente una naturalización de la reflexión filosófica.

\section{Referencias}

Bunge, M., 1976, Tecnología y filosofía, Universidad Aut. de Nuevo León, Monterrey. Dewey, J., 1993, La reconstrucción de la filosofía, Planeta Agostini, Barcelona.

Fisher, J., 2010, El hombre y la técnica: hacia una filosofía política de la ciencia y la tecnología, UNAM, México.

Kelsen, H., 1995, ¿Qué es la justicia?, Fontamara, México.

Ortega y Gasset, J., 1982, Meditación de la técnica y otros ensayos sobre ciencia y filosofía, Alianza, Madrid.

Perelman, Ch., 1964, De la Justicia, Centro de Estudios Filosóficos, UNAM, México. Putnam, H., 1994, Cómo renovar la filosofía, Cátedra, Madrid.

${ }^{18}$ Fernando Broncano, en la línea de Habermas, sugiere este mismo papel de control de calidad para cierta idea de democracia. Creo sin embargo que si hay que llevar a cabo un control de calidad dentro de una teoría de la evaluación de la técnica, entonces tal control debe incluir entre sus objetos a la democracia misma. Es sólo mediante el ejercicio de la razón en y a través de la filosofía política que sería posible tal control o, si se quiere, meta-control de calidad sobre esa técnica instanciada en la política. 
Rescher, N., 1993, La racionalidad, Tecnos, Madrid.

Smith, G. B., 2007, "What is Political Philosophy? A Phenomenological View", en Perspectives on Political Science, vol. 36, no. 2, Heldref Publications, Filadelfia.

Recibido el 22 de mayo 2010 Aceptado el 17 de junio de 2010 